\title{
Fast performance evaluation of LoRa communications over Rayleigh fading channels
}

\author{
Jules Courjault \\ Univ Rennes, CNRS, IRISA \\ Lannion, France
}

\author{
Baptiste Vrigneau \\ Univ Rennes, CNRS, IRISA \\ Lannion, France
}

\author{
Olivier Berder \\ Univ Rennes, CNRS, IRISA \\ Lannion, France
}

\begin{abstract}
In the last years, LoRa has emerged as a high potential candidate among several standards for the Internet of Things (IoT) subject to an exponential development. LoRa modulation is based on a classical chirp spread-spectrum technique and permits wireless data transmission up to $50 \mathrm{kbps}$ over several kilometers with a high energy efficiency. Although a well-known principle, its performance in terms of symbol or bit error probability has been theoretically analyzed in few recent papers only. Recently, closedform approximations of Bit Error Probability (BEP) for additive white Gaussian noise channels and Rayleigh fading channels were proposed. In this paper, we introduce a new approach using Marcum function for approximating the LoRa BEP. The latter is available for both Additive White Gaussian Noise channels and Rayleigh fading channels and the approach should deal with a variety of fadings. Simulations and comparisons with the state of the art show that the proposed approximation is almost ten times more accurate and may be considered as a numerical reference.
\end{abstract}

Index Terms-LP-WAN and IoT, LoRa transmission, Binary Error Probability, Performance analysis, Marcum function

\section{INTRODUCTION}

As the cost of sensors and microcontrollers has severely decreased, the deployment of billions of sensing and communicating objects, giving birth to the Internet of Things (IoT), is now an evidence. This phenomenon allows various monitoring applications in very different domains, for instance, health of people or structures, smart cities or restricted areas surveillance... environmental monitoring or smart agriculture are typical applications that need long range transmissions but low energy consumption, since wireless nodes are sometimes deployed in harsh environments and it may be difficult or costly to change batteries [1]. Fortunately, new wireless solutions known as Low Power Wide Area Networks (LPWAN) [2] allows to answer these needs, proposing very interesting energy efficiency for data transmissions at dozens of kbps over several kilometers. LoRa technology, developed by Semtech [3] [4] and promoted by the LoRa Alliance, has already seduced many industrials due to the data rates it can achieve while consuming very few energy. Using unlicensed bands $(433 \mathrm{MHz}$ and $868 \mathrm{MHz}$ or $915 \mathrm{MHz}$ depending on the countries), the LoRa physical layer is based on the Chirp Spread Spectrum (CSS) modulation with a linear variation of frequency on time [5] [6], which allows the reduction of both interference and Doppler effects [7]. One of the key points of LoRa is its adaptability thanks to three parameters that permit to configure the modulation: the bandwidth $B W$, the spreading factor $S F$, and the coding ratio $C R$.
However, while dramatically important before deployment to optimize networks coverage, the performance evaluation of LoRa is still an issue and rigorous theoretical studies of this technology are missing. Mathematical derivations for modulation and demodulation were proposed in [8] but the paper still lacks a theoretical analysis of the Bit Error Probability (BEP), as stated in [9]. In fact, the theoretical expression is known for decades and was given in [10], but it is not really computable and there is a need for accurate approximations that would give a fast performance evaluation. A same particularity of the available expressions is the use of the complementary error function, and the novelty of this work is to provide an accurate approximation based on the Marcum function [11] which was already employed in communications theory [12]. This new approach is applied to the BEP for an Additive White Gaussian Noise (AWGN) channel and next to Rayleigh fading channels.

The rest of the paper is organized as follows: Section II presents the state of the art and the required basics. Section III explains how Marcum function can lead to an accurate BEP approximation. Section IV compares results to the state of the art on fading channels before the conclusion is drawn in Section V.

\section{State OF The ART}

\section{A. LoRa BEP theoretical expression and issues}

For conciseness, only the needed equations are introduced and the reader can refer to [8] and [9] for detailed explanations. We remind that the receiver first applies a down chirp, secondly performs the discrete Fourier transform of the receive signal, and third estimates the symbols with the maximum value criterion. As the symbols have the same probability, the study can focus on the probability of correct decision of the symbol $m$, denoted $\overline{\mathbb{P}}_{m}$. According to [9], [10], this probability can be expressed as follows:

$$
\overline{\mathbb{P}}_{m}=\left(1-\exp \left(-\frac{\left|\sqrt{N}+W_{p}[N-m]\right|^{2}}{\sigma^{2}}\right)\right)^{N-1} .
$$

where $N=2^{S F}, S F$ is the spreading factor and is an integer between 7 and 12, $W_{p}[N-m]$ is a Gaussian complex noise with variance $\sigma^{2}$. By defining the Signal-to-Noise Ratio $\mathrm{SNR}=1 / \sigma^{2}$ and the random variable

$$
Z=\frac{\left|\sqrt{N}+W_{p}[N-m]\right|^{2}}{\sigma^{2}},
$$


$Z$ follows a noncentral $\chi^{2}$ law with 2 degrees of freedom and the noncentrality parameter $\lambda=2 N \cdot$ SNR. Its probability density function (pdf) is expressed as:

$$
f_{Z}(x)=\frac{1}{2} \exp \left(-\frac{x+\lambda}{2}\right) I_{0}(\sqrt{\lambda x}),
$$

with $I_{0}($.$) the modified Bessel function of the first kind of$ order 0. Finally, the last step is the computation of the average Symbol Error Probability (SEP):

$$
\begin{aligned}
\mathrm{SEP} & =\int_{0}^{+\infty}\left(1-\left(1-e^{-z / 2}\right)^{N-1}\right) f_{Z}(z) d z, \\
& =\frac{1}{N} \sum_{k=1}^{N-1} C_{N}^{k+1}(-1)^{k+1} e^{-N \cdot \operatorname{SNR} \frac{k}{(k+1)}},
\end{aligned}
$$

with $C_{N}^{k+1}=N ! /(k+1) !(N-k-1)$ ! the binomial coefficient. However, the numerical computation of this result suffers from accuracy issue due to the high value of the binomial coefficient for $S F \geq 7$, thus the results of (5) can not be obtained numerically. This problem was already pointed out in [9], [10], and justifies the crucial need for finding accurate approximations. As example, authors in [13] used Monte Carlo simulations to average and obtain the BER.

\section{B. Existing approximations of the BEP}

Generally, the performance is expressed with the BEP but the SEP can be used to estimate it by using the following relation [10]:

$$
\mathrm{BEP}=\frac{2^{S F-1}}{2^{S F}-1} \mathrm{SEP} .
$$

Reynders et al. proposed in [14] an approximation based on the Q-function or the tail distribution of the standard normal distribution. Recently, the authors in [9] proposed two new accurate estimations of the BEP for LoRa communications in AWGN and Rayleigh fading channels. The principle is to approximate a Ricean distribution by a Gaussian one and the expression for AWGN channel is based on two Q-functions using the $m^{\text {th }}$ harmonic number. As far as we know, the only result about LoRa performance in a Rayleigh fading channel is also based on the Q-function [9]:

$$
\begin{array}{r}
\mathrm{BEP} \approx 0.5 \cdot\left(Q\left(-\sqrt{2 H_{N-1}}\right)-\right. \\
\left.\sqrt{\frac{N \mathrm{NNR}}{N \mathrm{SNR}+1}} e^{\frac{H_{N-1}}{N \mathrm{SNR}+1}} Q\left(-\sqrt{2 H_{N-1} \frac{N \mathrm{NNR}}{N \mathrm{SNR}+1}}\right)\right)
\end{array}
$$

where $H_{m}$ is the harmonic number defined above.

\section{NEW METHOD BASED ON MARCUM FUNCTION}

\section{A. Considering $g(z)$ for approximating $\overline{\mathbb{P}}_{m}$}

The results introduced hereabove attempt to transform noncentral Chi squared or Ricean distributions into Gaussianbased approximations. The novelty of the proposed work is to focus on the expression $g(z)=1-\left(1-e^{-z / 2}\right)^{N-1}$ while keeping the noncentral Chi squared distribution. When $g(z)$ is extended in a sum, the more $k$ and $z$ increase, the less significant the term $e^{-k z / 2}$ is. Thus, the sum can be limited to

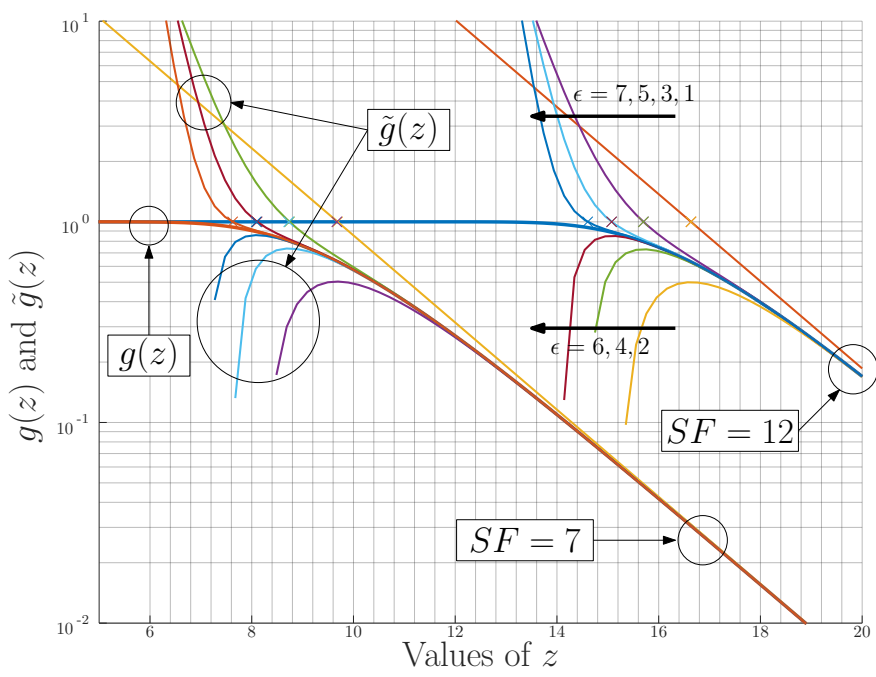

Fig. 1. Comparison of the exact function $g(z)$ and approximations with different orders $\epsilon$ from 1 to 7 and $S F=7,12$. Values of $z_{c}$ are plotted with cross marker.

$\epsilon$ terms, which is equivalent to find the approximation thanks to the Taylor's series for high values of $z$. The first main expression is:

$$
g(z) \approx \tilde{g}(z)=\sum_{k=1}^{\epsilon}(-1)^{k+1} C_{N-1}^{k} e^{-k z / 2} .
$$

Fig. 1 shows the comparison with the exact $g(z)$ and different approximation orders, $\epsilon=1$ to 7 . The good point is that binomial coefficients are limited to low values of $k$, which solves a computational issue. Since $N$ is high, especially for $S F=12$, the binomial coefficient $C_{N-1}^{k}$ will suffer from loss of precision, typically when $k$ is superior to 5 . The drawback is although the approximation is tightly close for high values of $z$, the divergence is problematic: the approximation tends toward infinity for odd values of $\epsilon$ and toward zero for even orders. Nevertheless, a simple and efficient approximation based on a piecewise definition is obvious in order to obtain the initial value 1 :

$$
g(z) \approx\left\{\begin{array}{cc}
1 & \text { if } z \leq z_{c} \\
\tilde{g}(z) & \text { if } z>z_{c}
\end{array} .\right.
$$

where $z_{c}$ is a threshold depending on the values of $\epsilon$ and $S F$. The different values of $z_{c}$ are available in the Fig. 1 and a first quick observation is that the piecewise approximation should be accurate for low values of $\epsilon$. Finding the values of $z_{c}$ is equivalent to find the roots of an $\epsilon$-order polynom $\tilde{g}(z)=1$ after applied the variable change $X=e^{-\frac{z}{2}}$ in (8). Since the approximation with even values of $\epsilon$ can not reach the value 1 , the study is limited to odd value cases. The only known assumption is that $z_{c}$ is the unique positive real root. However, we are able to obtain the exact solutions for $\epsilon=1$ and $\epsilon=3$. By using the notation $z_{c}(\epsilon)$, we have:

$$
\begin{array}{rlrl}
z_{c}(1) & = & & 2 \log (N-1) \\
z_{c}(3) & = & -2 \log \left(\tau-\frac{N-4}{(N-2)(N-3)^{2}} \frac{1}{\tau}+\frac{1}{N-3}\right) \\
\tau & =\left(\frac{(N-4)(N-5)}{(N-1)(N-2)(N-3)^{3}}-\frac{\sqrt{2}(N-4)}{(N-1)(N-2)^{1.5}(N-3)^{1.5}}\right)^{\frac{1}{3}}
\end{array}
$$


As two exact roots are available and after a straightforward numerical observation, we propose a polynomial function of order 1 that gives the exact values of $z_{c}$ for $\epsilon=1, \epsilon=3$ and an approximation for higher values:

$$
\begin{aligned}
z_{c}(\epsilon) & =-2 \log \left(\alpha_{1} \epsilon+\alpha_{0}\right) \\
\alpha_{0} & =\left(3 e^{-z_{c}(1) / 2}-e^{-z_{c}(3) / 2}\right) / 2 \\
\alpha_{1} & =\left(e^{-z_{c}(3) / 2}-e^{-z_{c}(1) / 2}\right) / 2
\end{aligned}
$$

The next step is to compute the expectation of $1-\overline{\mathbb{P}}_{m}$ by using the approximation of $g(z)$ :

$$
\begin{aligned}
\operatorname{SEP} \approx & \int_{0}^{z_{c}} f_{Z}(z) d z+ \\
& \int_{z_{c}}^{\infty} \sum_{k=1}^{\epsilon}(-1)^{k+1} C_{N-1}^{k} e^{-k z / 2} f_{Z}(z) d z .
\end{aligned}
$$

By using the definition of the pdf in (3), the SEP can be expressed by this practical result after some straightforward mathematical manipulations:

$$
\begin{array}{r}
\mathrm{SEP} \\
\frac{1}{N} \sum_{k=1}^{\epsilon+1} C_{N}^{k}(-1)^{k} e^{-\frac{N \cdot \mathrm{SNR}(k-1)}{k}} Q_{1}\left(\sqrt{\frac{2 N}{k} \mathrm{SNR}}, \sqrt{k z_{c}}\right),
\end{array}
$$

with $Q_{1}$ the Marcum function of order 1 defined by:

$$
Q_{M}(a, b)=\int_{b}^{\infty} x\left(\frac{x}{a}\right)^{M-1} e^{-\frac{x^{2}+a^{2}}{2}} I_{M-1}(a x) d x .
$$

\section{B. Application to the Rayleigh fading}

Let assume now a fading gain associated with an AWGN channel. The received signal is expressed as:

$$
r(t)=h s(t)+w(t)
$$

where $h$ is the channel fading gain, $s(t)$ is the LoRa chirp, and $w$ is an additive Gaussian noise. The term $h$ is an iid complex random variable and we assume that the channel is flat fading during the LoRa transmission, i.e., $h$ is constant for a full frame and will change for the next one. After the demodulation process, by using the linearity property of the Fourier transform, the signal obtained is:

$$
R_{p}[k]=h \sqrt{N} \delta(k+m-N)+W_{p}[k]
$$

Thus, the probability $\overline{\mathbb{P}}_{m}$ according to the value $h$ that the right symbol is decoded is expressed as:

$$
\begin{aligned}
\overline{\mathbb{P}}_{m} & =\operatorname{Pr}\left[\forall k \neq N-m:\left|R_{p}[k]\right|^{2}<\left|R_{p}[N-m]\right|^{2}\right] \\
& =\prod_{\substack{k=0 \\
k \neq N}}^{N-m} \operatorname{Pr}\left[\left|W_{p}[k]\right|^{2}<\left|h \sqrt{N}+W_{p}[N-m]\right|^{2}\right] \\
& =F_{\chi_{2}^{2}}\left(\frac{\left|h \sqrt{N}+W_{p}[N-m]\right|^{2}}{\sigma_{n}^{2}}\right)^{N-1}
\end{aligned}
$$

As described above for the Gaussian case, we obtain

$$
\overline{\mathbb{P}}_{m}=\left(1-\exp \left(-\frac{\left|h \sqrt{N}+W_{p}[N-m]\right|^{2}}{\sigma_{n}^{2}}\right)\right)^{N-1} \text {. }
$$

Let's denote the variable $Z$ as:

$$
Z=\mathrm{SNR} \cdot\left|h \sqrt{N}+W_{p}[N-m]\right|^{2} .
$$

The random variable $Z$ follows then a noncentral chi-squared law with 2 degrees of freedom with the noncentral parameter $\lambda=\operatorname{SNR} \cdot 2|h|^{2} N$. The link between SEP with a fading gain and AWGN case from eq. (17) is then:

$$
\left.\operatorname{SEP}(\mathrm{SNR})\right|_{h}=\operatorname{SEP}\left(|h|^{2} \mathrm{SNR}\right) .
$$

In order to obtain the SEP, the distribution of $h$ must be defined and the average computed. In this paper, we apply for the Rayleigh case:

$$
\operatorname{SEP}_{h}(\mathrm{SNR})=\int_{0}^{+\infty} \operatorname{SEP}(x \operatorname{SNR}) f_{|h|^{2}}(x) d x,
$$

where $f_{|h|^{2}}(x)$ is the pdf of the squared modulus of $h$. The previous result based on Marcum function will be used and the difficulty is to deal with it in the integral.

In the Rayleigh case, $h$ is a complex random Gaussian variable with zero mean and unit variance. The squared modulus is well-known and the SEP can be obtained by computing:

$$
\operatorname{SEP}_{\text {Ray }}(\operatorname{SNR})=\int_{0}^{\infty} \operatorname{SEP}(x \operatorname{SNR}) e^{-x} d x .
$$

By using eq. (17), the new approximation is:

$$
\begin{array}{r}
\operatorname{SEP}_{\text {Ray }}(\mathrm{SNR}) \approx 1+\frac{1}{N} \sum_{k=1}^{\epsilon+1} C_{N}^{k}(-1)^{k} \times \\
\int_{0}^{\infty} e^{-\left(\frac{N \cdot \mathrm{SNR}(k-1)}{k}+1\right) x} Q_{1}\left(\sqrt{\frac{2 N}{k} x \mathrm{SNR}}, \sqrt{k z_{c}}\right) d x .
\end{array}
$$

Authors in [15] focused on some integrals involving Marcum function and especially the expression $\mathcal{F}(l, m, a, b, p)$ defined as:

$$
\mathcal{F}(l, m, a, b, p)=\int_{0}^{+\infty} x^{l-1} Q_{m}(a \sqrt{x}, b) e^{-p x} d x .
$$

In order to reuse the results, the $\mathrm{SEP}_{\text {Ray }}$ can be written as:

$$
\begin{array}{r}
\operatorname{SEP}_{\text {Ray }}(\mathrm{SNR}) \approx 1+\frac{1}{N} \sum_{k=1}^{\epsilon+1} C_{N}^{k}(-1)^{k} \times \\
\mathcal{F}\left(1,1, \sqrt{\frac{2 N}{k} \mathrm{SNR}}, \sqrt{k z_{c}}, \frac{(k-1) N}{k} \mathrm{SNR}+1\right) .
\end{array}
$$

The expression of $\mathcal{F}(l, m, a, b, p)$ is available for $l=1$ and $m=1$ [15, eq.(19)] and is easy to compute by using classical mathematical functions.

\section{High-SNR approximation}

This subsection provides a high-SNR approximation with the form $(c \mathrm{SNR})^{-o}$ where $c$ is the code gain and $o$ the diversity order. From [15, eq.(19)], the expression of $\mathcal{F}(k, m, a, b, p)$ can be simplified as:

$$
\mathcal{F}(1,1, a, b, p)=\frac{e^{-\frac{p b^{2}}{a^{2}+2 p}}}{p},
$$


and then

$$
\operatorname{SEP}_{\text {Ray }}(\mathrm{SNR}) \approx 1-\sum_{k=0}^{\epsilon} C_{N-1}^{k}(-1)^{k} \frac{e^{-\frac{(k N \mathrm{SNR}+k+1) z_{c}}{2 N \mathrm{NNR}+2}}}{k N \mathrm{SNR}+1} .
$$

Now, considering that SNR $\gg 1$, the SER can be approximated by:

$$
\begin{gathered}
\operatorname{SEP}_{\text {Ray }}(\mathrm{SNR}) \approx 1-e^{-\frac{z_{c}}{2 N \mathrm{SNR}}}- \\
(N \mathrm{SNR})^{-1} \sum_{k=1}^{\epsilon} \frac{C_{N-1}^{k}(-1)^{k} e^{-k \frac{z_{c}}{2}}}{k} .
\end{gathered}
$$

By applying the limited development of exponential around zero, the SER becomes:

$$
\begin{array}{r}
\operatorname{SEP}_{\text {Ray }}(\mathrm{SNR}) \approx \\
(N \mathrm{SNR})^{-1}\left(\frac{z_{c}}{2}-\sum_{k=1}^{\epsilon} \frac{C_{N-1}^{k}}{k}(-1)^{k} e^{-k \frac{z_{c}}{2}}\right) .
\end{array}
$$

It is obvious that the diversity order is 1 . As we will observe, the approximation converges quickly and the minimum order $\epsilon=1$ is enough accurate. Thus, by using $z_{c}=2 \log (N-1)$ and eq.(6), the BER at high SNR is simply expressed by:

$$
\mathrm{BEP}_{\text {Ray }}(\mathrm{SNR}) \approx(N \mathrm{SNR})^{-1}(\log (N-1)+1) .
$$

\section{RESULTS AND COMPARISONS}

\section{A. Impact of the order $\epsilon$ : accuracy and value choice}

In order to evaluate the convergence and the accuracy according to the order $\epsilon$, a convergence parameter is defined by:

$$
\Delta_{\mathrm{BEP}, i \rightarrow j}=\left(\left.\mathrm{BEP}\right|_{\epsilon=i}-\left.\mathrm{BEP}\right|_{\epsilon=j}\right) /\left.\mathrm{BEP}\right|_{\epsilon=i} .
$$

It allows the evaluation of the BEP variation when the order $\epsilon$ is increased. Fig. 2 shows three cases $\Delta_{\mathrm{BEP}, 1 \rightarrow 3}, \Delta_{\mathrm{BEP}, 3 \rightarrow 5}$, and $\Delta_{\mathrm{BEP}, 5 \rightarrow 7}$ as a function of SNR for different values of $S F$. The behavior is an increasing error but is constant at high SNR, i.e., diversity orders are equal but the code gain has a constant error. The variation when the order $\epsilon$ increases from 1 to 3 is inferior to $5 \%$, the variation is less than $2 \%$ when the order $\epsilon$ changes from 3 to 5 , and less than $0.6 \%$ for the next step 5 to 7 . As a conclusion, the approximation converges very fast: the order $\epsilon=1$ is already a good approximation with the best trade-off between complexity and accuracy, and it is not necessary to increase $\epsilon$ higher than 3 .

\section{B. BEP comparisons}

Fig. 3 compares the proposed approximation with simulated BEP, obtained with Monte-Carlo simulations, and the results of [9, eq.(33)] which is the best of the state of the art. These simulations validate that the Marcum-based approximation is tightly accurate with an order lower than $\epsilon=5$. Compared to [9, eq.(33)], our proposed solution $i$ ) is more accurate, ii) is not impacted by the value of $S F$, and iii) has a simpler mathematical expression. In order to highlight these differences, Fig. 4 proposes to focus on the relative error of approximations with respect to the simulated BEP. The approximation [9, eq.(33)] is compared to our proposed
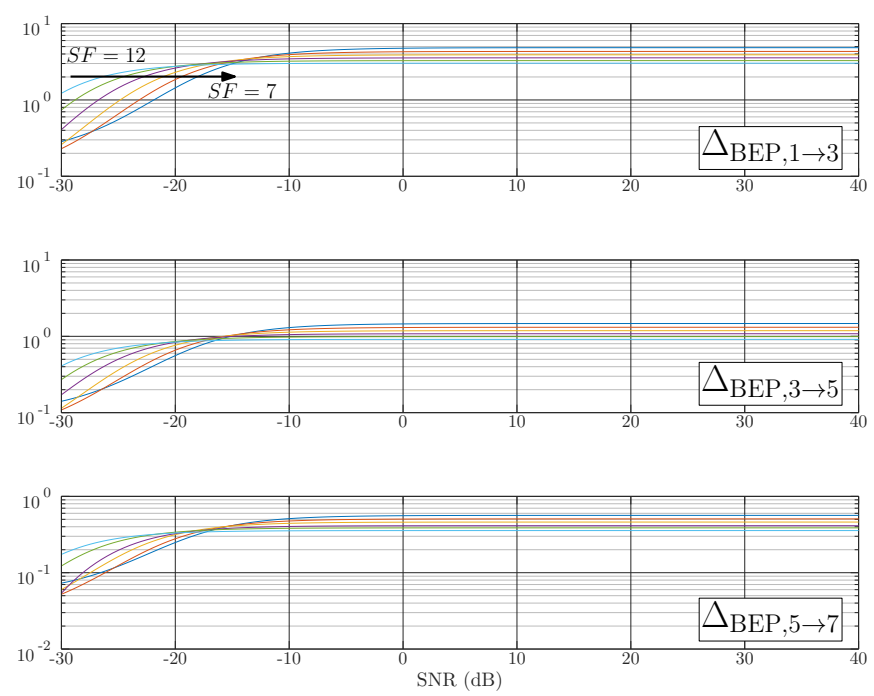

Fig. 2. Accuracy loss when increasing approximation order $\Delta_{\mathrm{BEP}, 1 \rightarrow 3}$, $\Delta_{\mathrm{BEP}, 3 \rightarrow 5}$, and $\Delta_{\mathrm{BEP}, 5 \rightarrow 7}$ as a function of SNR for $S F=7$ to 12 .

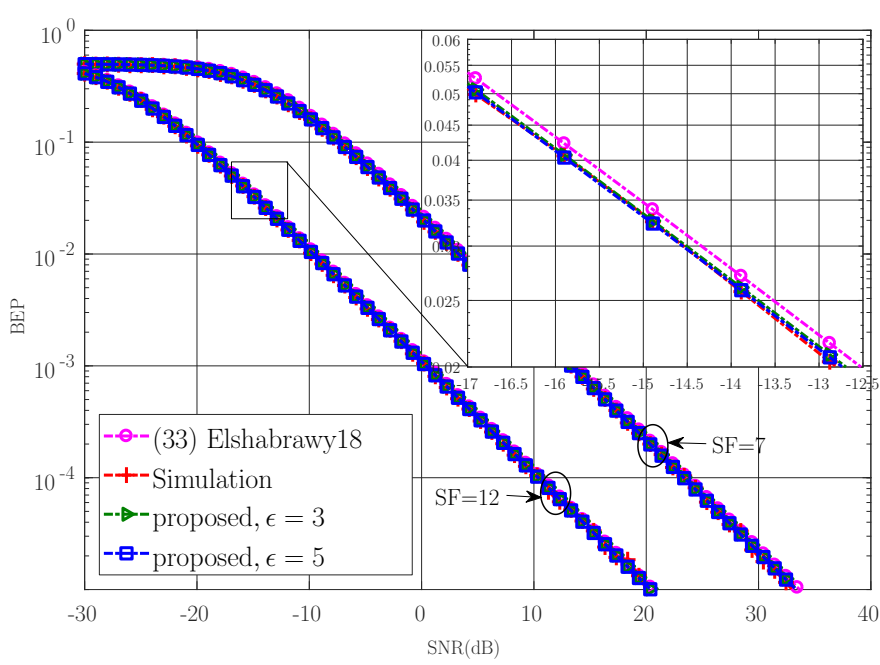

Fig. 3. BEP of the proposed approximation with the order $\epsilon=\{3,5\}$, simulation, and approximation in [9] for $S F=7$ to 12 .

solution with $\epsilon=3,5$. The observation of the curves confirms that the Marcum-based expression is more accurate than $[9$, eq.(33)] and increasing the order $\epsilon$ enhances the accuracy. At $\mathrm{SNR}=-15 \mathrm{~dB}$, the approximation error for a spreading factor of 7 is about $1 \%$, which represents a gain of almost 10 compare to previous works.

\section{BEP high-SNR approximation}

Fig. 5 plots the proposed BER approximation with $\epsilon=7$ and the high-SNR approximation with $\epsilon=1$ defined in eq.(34) for $S F=7$ to 12 . As mentioned above, our proposed approximation with $\epsilon=7$ is accurate and can serve as a reference instead of simulations. The comparisons show that for a BEP inferior to $10^{-2}$, the approximation is accurate enough and is mingled with the other curve. 


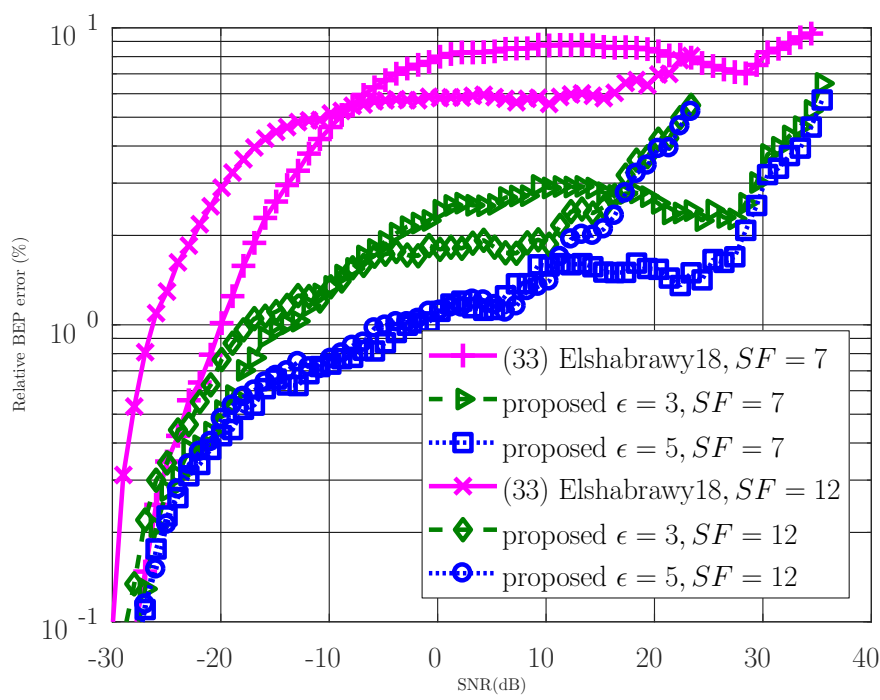

Fig. 4. Relative error between the proposed approximation and [9] with respect to the simulation for $\epsilon=3,5$ and $S F=7$ to 12 .

\section{CONCLUSion}

In this paper, a new approximation of BEP for LoRa transmissions is proposed for a Rayleigh fading channel. Instead of approximating a Ricean or noncentral Chi squared distribution thanks to a Gaussian one, we proposed to associate this distribution with a piece-wise approximation of the expression to average. It leads to a result using the Marcum function. Simulation results confirm that the proposed solution is more accurate than the state-of-the-art for a huge SNR range and may be considered, to the best of the authors' knowledge, as the best numerical reference for the LoRa BEP. The Marcum function is a well-known function and was already used in communication theory. As a lot of results are available in scientific literature, the proposed approach is a promising solution to deal with open problems for LoRa theoretical performance study such as other fading channels, synchronization error, or mobility impact.

\section{ACKNOWLEDGMENT}

This work was supported by the ALAMO project founded by the competitiveness Pole "Image and Networks" of Brittany region.

\section{REFERENCES}

[1] F. Ait Aoudia, M. Gautier, M. Magno, M. Le Gentil, O. Berder, and L. Benini, "Long-Short Range Communication Network Leveraging LoRa and Wake-up Receiver," Microprocessors and Microsystems: Embedded Hardware Design (MICPRO), vol. 56, pp. 184 - 192, December 2017.

[2] R. Sanchez-Iborra and M. Cano, "State of the art in LP-WAN solutions for industrial IoT services," Sensors, vol. 16, no. 5, p. 708, 2016. [Online]. Available: https://doi.org/10.3390/s16050708

[3] O. Seller and N. Sornin, "Low power long range transmitter," Aug. 2014, US Patent App. 14/170,170. [Online]. Available: https://www.google.com/patents/US20140219329

[4] Semtech, "SX1272/73-860 MHz to $1020 \mathrm{MHz}$ low power long range transceiver," Tech. Rep. Datasheet, Mar. 2015. [Online]. Available: http://www.semtech.com/images/datasheet/sx1272.pdf

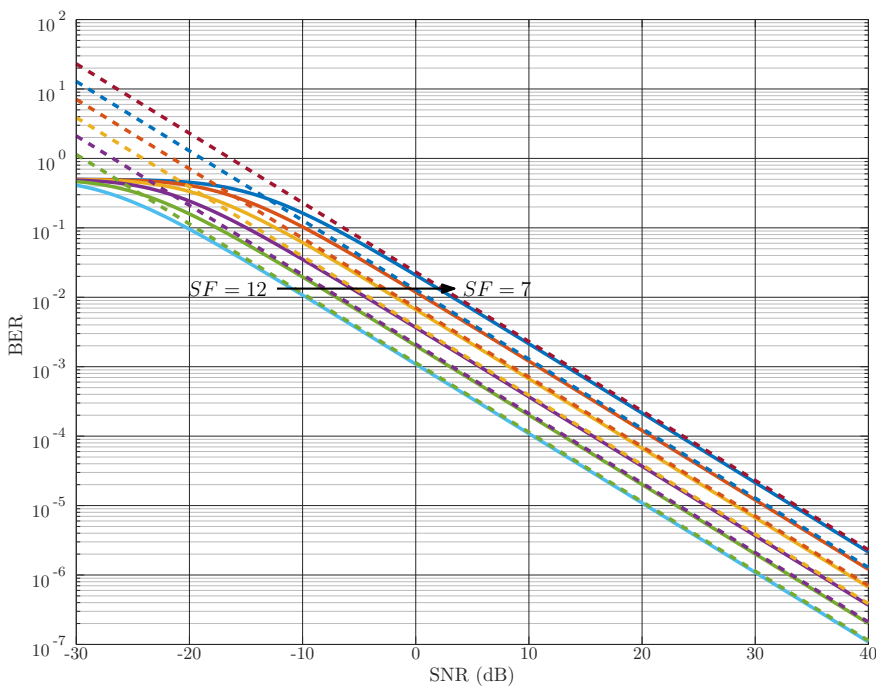

Fig. 5. High-SNR approximation for a Rayleigh fading for $S F=7$ to 12 : line is proposed approximation with $\epsilon=7$ and dashed-line are high-SNR approximation.

[5] A. Springer, W. Gugler, M. Huemer, L. Reindl, C. C. W. Ruppel, and R. Weigel, "Spread spectrum communications using chirp signals," in IEEE/AFCEA EUROCOMM 2000, Information Systems for Enhanced Public Safety and Security, 2000, pp. 166-170.

[6] B. Reynders and S. Pollin, "Chirp spread spectrum as a modulation technique for long range communication," in 2016 SCVT, Nov. 2016, pp. 1-5.

[7] X. Le, B. Vrigneau, M. Gautier, M. Mabon, and O. Berder, "Energy/reliability trade-off of LoRa communications over fading channels," in 2018 25th International Conference on Telecommunications (ICT), June 2018, pp. 544-548.

[8] L. Vangelista, "Frequency shift chirp modulation: The lora modulation," IEEE Signal Processing Letters, vol. 24, no. 12, pp. 1818-1821, Dec 2017.

[9] T. Elshabrawy and J. Robert, "Closed form approximation of LoRa modulation BER performance," IEEE Communications Letters, vol. 22 no. 9, pp. 1778-1781, 2018.

[10] J. G. Proakis, Digital Communications, 5th edition, N. York:McGrawHill, Ed., 1995.

[11] J. I. Marcum, Table of Q functions, R. Corporation, Ed., Jan. 1950.

[12] M. K. Simon and M. . Alouini, "Exponential-type bounds on the generalized Marcum Q-function with application to error probability analysis over fading channels," IEEE Transactions on Communications, vol. 48, no. 3, pp. 359-366, March 2000.

[13] G. Ferré and A. Giremus, "Lora physical layer principle and performance analysis," in 2018 25th IEEE International Conference on Electronics, Circuits and Systems (ICECS), Dec 2018, pp. 65-68.

[14] B. Reynders, W. Meert, and S. Pollin, "Range and coexistence analysis of long range unlicensed communication," in The 23rd International Conference on Telecommunications (ICT), May 2016, pp. 1-6.

[15] P. C. Sofotasios, S. Muhaidat, G. K. Karagiannidis, and B. S. Sharif, "Solutions to integrals involving the Marcum $Q$-function and applications," IEEE Signal Processing Letters, vol. 22, no. 10, pp. 1752-1756, Oct 2015 . 